\title{
Management of hemostasis in the perioperative period: the anesthesiologist's perspective
}

\author{
Jean-François Hardy MD, ${ }^{*}$ Brigitte Jude MD PhD, $†$ Charles Marc Samama MD PhD $\ddagger$
}

A $s$ medical students, we shudder when the words «coagulation cascade» are pronounced by a teacher or invited lecturer. For a majority of clinicians, this resentment for all that is related to «coagulation» persists through their professional lives. Yet, some of us, like anesthesiologists and surgeons, must be sufficiently knowledgeable in matters related to hemostasis and thrombosis to deal with relatively common problems likely to occur when the hemostatic system is breached/stressed during the course of surgery. While bleeding is a major concern during and immediately after the operation, surgery induces a hypercoagulable state and thrombosis will, later, become a concern.

So, what should we, as anesthesiologists, know about hemostasis and thrombosis? We cannot, obviously, be expected to be experts in the field: hematologists are the experts and we rely on their assistance when the riding gets rough. On the other hand, we must know enough to be able to manage the most common disorders of hemostasis related to surgery and trauma. We must understand the basic concepts underlying the physiology of hemostasis and what the tests tell us (or don't). Hence, this Supplement to the Canadian Journal of Anesthesia was prepared by the Groupe d'Intérêt en Hémostase Périopératoire (GIHP or, in English, Perioperative Hemostasis Interest Group).

In essence, hemostasis is the process by which the body stops bleeding when the vascular endothelium is disrupted. When collagen is exposed, platelets react to release a number of mediators that will 1 ) induce vasoconstriction at the site of injury; 2) stimulate platelet aggregation; and 3) trigger the coagulation cascade. Vasoconstriction reduces blood flow at the site of injury, platelet aggregation yields the primary hemostatic plug, and coagulation results in the generation of thrombin that converts fibrinogen into fibrin. The latter stabilizes the platelet plug. The first section of the Supplement describes normal/pathological hemostasis and its assessment in the laboratory or at the patient's bedside.

The second section of the Supplement describes how anesthesiologists manage the risk of bleeding in the perioperative period. Hemostasis can be disrupted in a number of clinical situations involving, for example, cardiopulmonary bypass, hepatic disease, trauma, etc. Colloids administered to maintain normovolemia can affect hemostasis. Major bleeding will, in itself, impair hemostasis as red cells, platelets and coagulation factors are depleted. Pharmacological agents are available to reduce blood losses and transfusions in the surgical patient. Anesthesiologists must be knowledgeable in their use, indications and contraindications.

After surgery, once the risk of hemorrhage has been controlled, hypercoagulability can be responsible for major adverse events such as deep vein thrombosis and pulmonary embolism. The third section of the Supplement describes how thrombotic events can be minimized and/or treated. A specific article is devoted to perioperative antithrombotic therapy in cardiac surgery.

Finally, nowadays, more and more patients are coming to surgery while receiving antithrombotic therapy whether with antiplatelet agents or vitamin $\mathrm{K}$ antagonists. The fourth section of the Supplement describes the management of these patients and that of a major consequence of heparin anticoagulation: heparin induced thrombocytopenia.

Obviously, the Supplement put together by the GIHP is not a textbook on hemostasis and thrombosis. Neither is it complete. However, we hope the series of articles will assist anesthesiologists in overcoming their fundamental aversion for the subject, and assist them in their daily clinical practice.

From the Department of Anesthesiology, ${ }^{*}$ Hôpital Notre-Dame, Centre Hospitalier de l'Université de Montréal, Montréal, Québec, Canada; the Institut d'Hématologie-Transfusion, $†$ Université de Lille, Lille, France; and the Department of Anesthésie-Réanimation, $\ddagger$ Assistance Publique-Hôpitaux de Paris, Centre Hospitalier Universitaire Avicenne, Bobigny, France.

Address correspondence to: Dr. Jean-François Hardy, Département d'anesthésiologie, CHUM Hôpital Notre-Dame, Porte AS-1115-3, Pavillon Lachapelle, 1560, rue Sherbrooke est, Montréal, Québec H2L 4M1, Canada. E-mail: jean-francois.hardy@umontreal.ca 